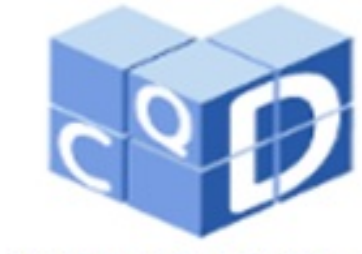

Revista Eletrônica

Paulista de Matemática

ISSN 2316-9664

Volume 7, dez. 2016

Edição ERMAC

Alexandre Calligaris Simões

Cotil - UNICAMP - Limeira

alexcsimoes@gmail.com

Renata Zotin Gomes de Oliveira

DM- IGCE - UNESP - Rio

Claro

rzotin@rc.unesp.br

\section{Usando o Geogebra no cálculo de área sob gráfico de funções no Ensino Médio}

Using Geogebra in area calculation under function graph in High School

\section{Resumo}

Este trabalho, que é parte da dissertação desenvolvida durante o Mestrado Profissional em Matemática em Rede Nacional (PROFMAT), tem como objetivo principal apresentar uma sugestão de como calcular áreas delimitadas por gráficos de funções para estudantes de Ensino Médio, utilizando o software Geogebra. Serão apresentadas algumas atividades que exemplificam o desenvolvimento da proposta e que poderiam ser desenvolvidas em conjunto com alguns conteúdos de Física, como por exemplo, cálculo de trabalho realizado por uma força ou espaço percorrido por um objeto.

Palavras-chave: Área; Soma de Riemann; Geometria Dinâmica.

\begin{abstract}
This work, which is part of a master thesis of PROFMAT, aims to present a suggestion of calculating areas bounded by graphs of functions to High school students using the Geogebra software. It will be presented some activities that exemplify the development of the proposal and it that could be developed with some contents of Physics, such as example, the work done by a force of magnitude $\mathrm{F}$ on a point that moves a displacement (not distance) $\mathrm{s}$ in the direction of the force.
\end{abstract}

Keywords: Area; Riemann Sum; Dynamic Geometry. 


\section{Introdução}

Calcular o valor da área de uma figura plana T é medir a região delimitada por esta, no plano. Para isso, precisamos definir uma unidade de área e compará-la com a região da figura. Logo, o resultado será um número que represente quantas vezes a unidade de área está contida na figura T. Em Lima(2004) apresenta-se de forma detalhada como se define área e a fórmula da área de figuras conhecidas.

Considerando o prévio conhecimento de como calcular a área de triângulos e retângulos, vejamos como determinamos a área de um polígono e de regiões delimitadas por curvas.

\section{Calculando área de figuras poligonais}

Em figuras poligonais, onde seus lados são segmentos de reta, não há dificuldade de calcular sua área pois qualquer figura poligonal, por mais irregular que seja, pode ser coberta com retângulos e triângulos justapostos (LIMA, 2004). Com isso, sua área terá o valor do somatório das áreas destas figuras. Vejamos um exemplo.

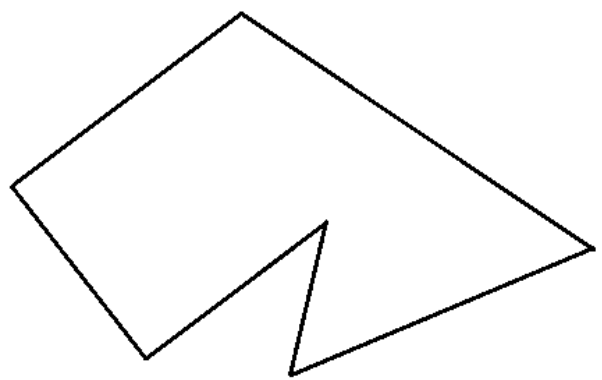

Figura 1: Polígono irregular.

O polígono da Figura 1 é facilmente particionado em retângulos e triângulos, como pode ser observado na Figura 2, através das subfiguras I, II, III, IV, justapostas.

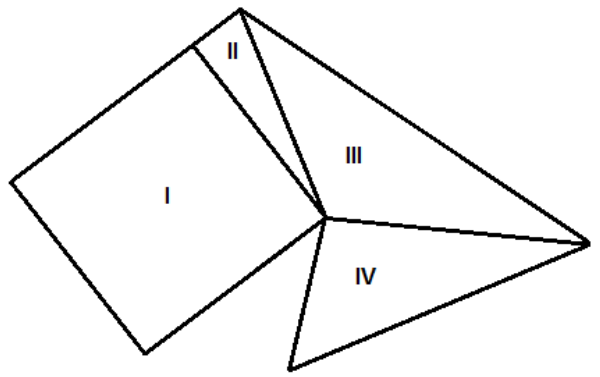

Figura 2: Polígono irregular particionado.

Então, para calcular sua área, basta encontrar a área das partes e somá-las. Temos, com isso, uma definição geral de área de um polígono. 
Podemos associar a cada polígono $P$ um número real não negativo, chamando a área de $P$, com as seguintes propriedades:

1. Polígonos congruentes têm áreas congruentes;

2. Se $P$ é um quadrado com lado unitário, então área de P é 1;

3. Se $P$ pode ser decomposto como reunião de $n$ polígonos $P_{1}, \ldots, P_{n}$, sendo que dois quaisquer deles têm em comum, no máximo, alguns lados, então a área de $P$ é a soma das áreas dos $P_{i}$.

Segue da última propriedade que se o polígono $P$ estiver contido no polígono $Q$ então a área de $P$ será menor do que a área de $Q$.

A Física no Ensino Médio contempla situações onde os valores de certas grandezas são equivalentes às áreas das figuras formadas sob uma curva, num determinado intervalo. Como exemplo, podemos citar o cálculo do trabalho realizado por uma força $F$ no deslocamento $d$ de um móvel sujeito à ação dessa força. Se um móvel tem um deslocamento de $5 \mathrm{~m}$ quando sofre a ação de uma força constante de $3 \mathrm{~N}$, o trabalho $(\tau)$ realizado por esta força é dado por $\tau=F . d=3.5=15 \mathrm{~J}$. Este resultado equivale à área sob a reta horizontal no intervalo entre $0 \mathrm{~m} \mathrm{e}$ 5m. (Figura 3).

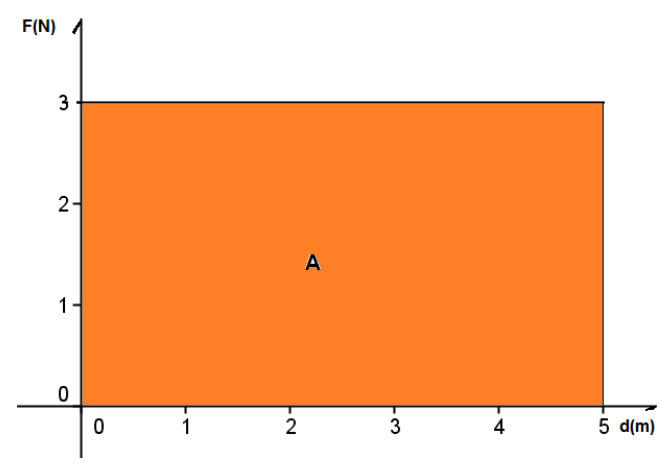

Figura 3: Exemplo de força constante.

No entanto, se um móvel, durante um deslocamento de $4 \mathrm{~m}$, sofre a ação de uma força que varia de acordo com o gráfico dado pela Figura 4, o trabalho realizado é dado por $\tau=\frac{(1+3) 4}{2}$.

Nestes exemplos temos, respectivamente, um retângulo e um trapézio determinados pelas forças. Nestas figuras simples, suas áreas determinam o valor da grandeza (trabalho) calculada, e podem ser determinadas através de fórmulas básicas já conhecidas desde o Ensino Fundamental.

\section{Calculando área de figuras não poligonais}

As propriedades citadas anteriormente são somente para polígonos e assim, não serviriam para encontrar, por exemplo, área do círculo e da elipse, pois não conseguimos compor perfeitamente suas regiões com triângulos e retângulos justapostos.

SIMÕES, A. C.; OLIVEIRA, R. Z .G. Usando o Geogebra no cálculo de área sob gráfico de funções no Ensino Médio. C. Q. D. - Revista Eletrônica Paulista de Matemática , Bauru, v. 7, p. 146-159, dez. 2016. Edição ERMAC. 


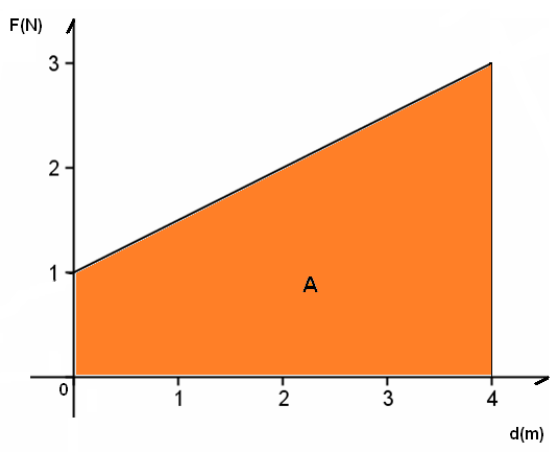

Figura 4: Exemplo de força variável.

Apresentaremos então uma estratégia para obtenção de áreas de figuras não poligonais. A área de uma figura plana $F$ arbitrária pode ser aproximada por um número real não negativo, que indicaremos como $a(F)$, que satisfaz

$$
a(P) \leq a(F) \leq a\left(P^{\prime}\right),
$$

onde $a(P)$ é a soma das áreas dos polígonos contidos em $F$ e $a\left(P^{\prime}\right)$ é a soma das áreas dos polígonos que contém $F$.

Vejamos exemplos que ilustram esta aproximação.

Para simplificar, mostraremos um exemplo considerando apenas polígonos retangulares contidos na região $F$ (Figura 5). Assim, com a reunião de suas áreas vamos obter uma aproximação da área de $F$, primeiramente, com uma aproximação por falta (Figura 5).

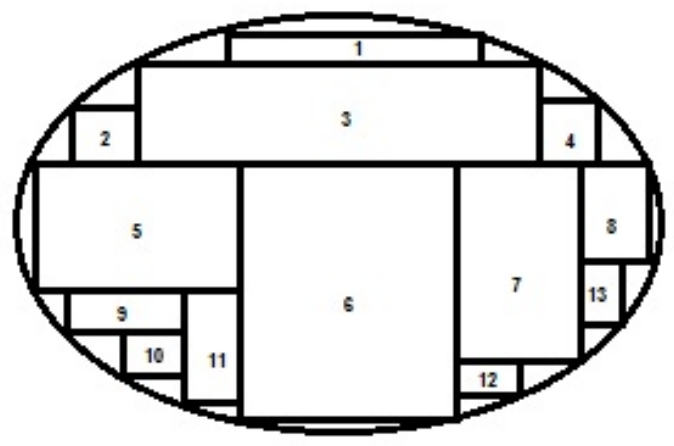

Figura 5: Aproximação da área por falta.

$$
A(F) \approx \sum_{j=1}^{13} A(j) .
$$

Num segundo exemplo, a região $F$ está contida na região formada pela reunião de retângulos. Agora, a área de $F$ é obtida por uma aproximação por excesso (Figura 6).

$$
A(F) \approx \sum_{j=1}^{16} A(j) .
$$




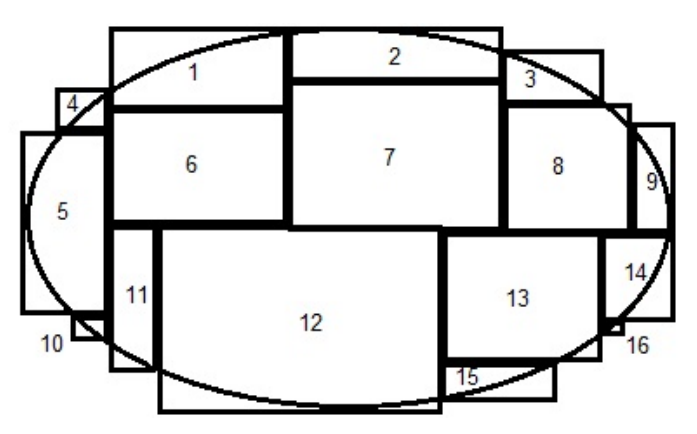

Figura 6: Aproximação da área por excesso.

Assim, uma estratégia para o cálculo da área de uma região fechada qualquer $(F)$ é obter uma aproximação através da soma das áreas de polígonos contidos em $F$ ou soma de áreas de polígonos que contém $F$. Usaremos essa mesma ideia na sugestão do cálculo de áreas delimitadas por gráfico de funções.

\section{1 Área sob gráfico de funções}

Se a força $F$ for dada por funções diferentes das apresentadas nos exemplos da seção anterior, a determinação do trabalho realizado envolve o cálculo da área delimitada por gráfico de função. Para tratarmos dessa situação, utilizaremos o software Geogebra, que permite uma visualização gráfica dinâmica do problema. Iniciaremos com um exemplo onde é possível comparar o valor exato da área da região com o valor aproximado obtido.

Consideremos um semicírculo de raio 2 (Figura 7), que também pode ser visto como o gráfico de $f(x)=\sqrt{4-x^{2}}$.

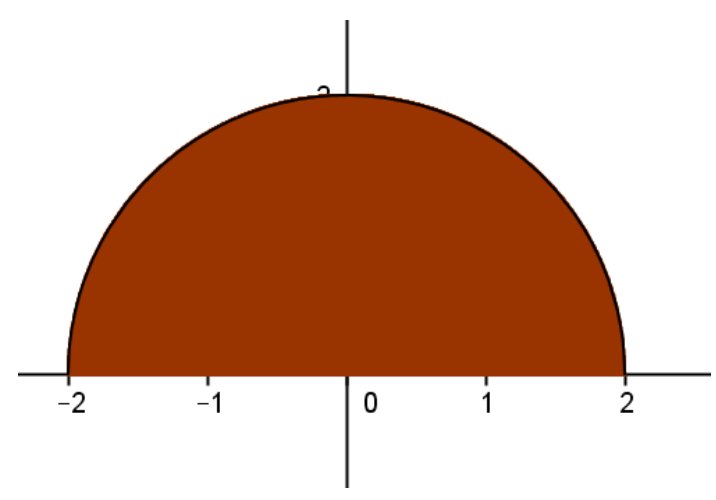

Figura 7: Área do semicírculo.

Inicialmente consideraremos retângulos justapostos, aproximando a área da região semicircular pela soma das áreas dos $n$ retângulos contidos nela (aproximação por falta). Para isso, tomaremos os subintervalos do intervalo $[-2,2]$ como base do retângulo e o valor mínimo da função em cada subintervalo como a altura dos retângulos. No caso da aproximação por excesso, a altura de cada retângulo será dada pelo valor máximo da função em cada subintervalo. 
Nestas aproximações, o Geogebra nos fornece gráficos dinâmicos e tabela de valores das áreas.

Quando a aproximação for por falta, a área obtida será denominada $A_{n i}$, onde $n$ indicará o número de subdivisões do intervalo e $i$ significa que é soma inferior.

$\mathrm{Na}$ aproximação por excesso, a área obtida será denominada $A_{n s}$, onde $n$ é o número de subdivisões do intervalo e $s$ indica soma superior.

Nas tabelas que serão apresentadas para cada etapa, a diferença entre o valor encontrado pela fórmula da área do círculo $(A=6,2832)$ e o valor $A_{n}$. Esta diferença é o erro ocorrido em cada etapa da aproximação.

Primeiramente, o desenvolvimento da aproximação por falta.

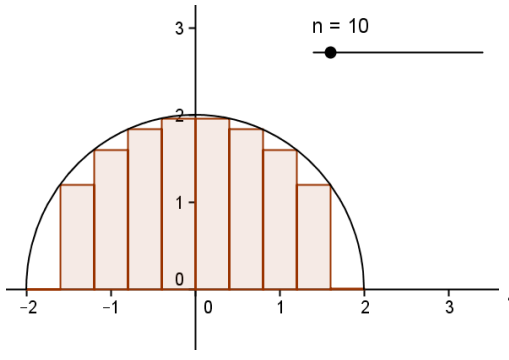

(a) $n=10$

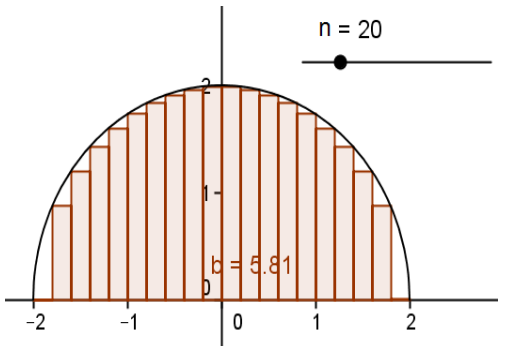

(b) $n=20$

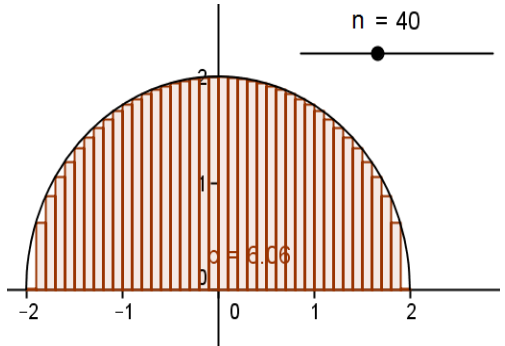

(c) $n=40$

Figura 8: Área por falta para alguns valores de $n$.

\begin{tabular}{|c|c|c|}
\hline$n$ & $A_{n i}$ & erro \\
\hline 10 & 5,2743 & 1,0089 \\
\hline 50 & 6,1045 & 0,1787 \\
\hline 100 & 6,1966 & 0,0866 \\
\hline 250 & 6,2495 & 0,03377 \\
\hline 530 & 6,2675 & 0,0157 \\
\hline 1100 & 6,2757 & 0,0075 \\
\hline 4500 & 6,2814 & 0,0018 \\
\hline 8700 & 6,2822 & 0,0001 \\
\hline
\end{tabular}

Tabela 1: Valores da área por falta, para alguns valores de $n$.

Para a aproximação por excesso, temos:

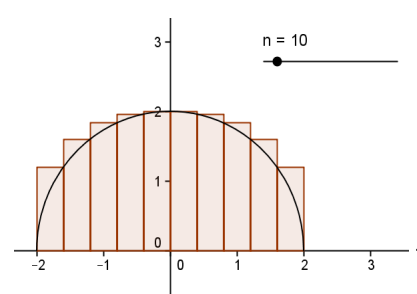

(a) $n=10$

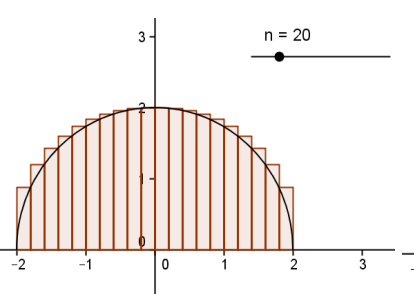

(b) $n=20$

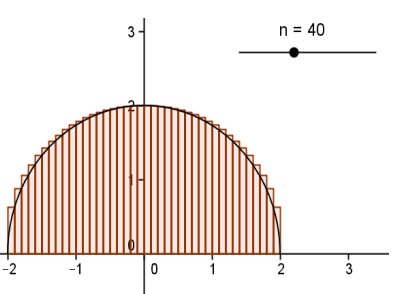

(c) $n=40$

Figura 9: Área por excesso, sob o gráfico da função da Figura 7, para alguns valores de $n$. 


\begin{tabular}{|c|c|c|}
\hline$n$ & $A_{n s}$ & erro \\
\hline 10 & 6,8741 & 0,5909 \\
\hline 50 & 6,4244 & 0,1412 \\
\hline 100 & 6,3565 & 0,0733 \\
\hline 250 & 6,3135 & 0,0303 \\
\hline 530 & 6,2977 & 0,0145 \\
\hline 1000 & 6,2910 & 0,0078 \\
\hline 6000 & 6,2845 & 0,0013 \\
\hline 9000 & 6,2840 & 0,0008 \\
\hline
\end{tabular}

Tabela 2: Valores da área por excesso, para alguns valores de $n$.

Podemos observar que, conforme aumentamos a quantidade de retângulos (consequentemente as bases são cada vez menores), a área da soma dos retângulos vai se aproximando da área do semicírculo, tanto na soma inferior como na superior. A coluna erro facilita a observação apresentando valores tendendo a zero.

Neste exemplo, como a figura é conhecida, ou seja, temos uma fórmula para calcular sua área na qual os alunos deste nível escolar já viram, podemos utilizá-lo, de modo comparativo, para dar sentido ao valor encontrado com a fórmula, ou, como exemplo inicial, demonstrar que este recurso gráfico nos fornece resultados verdadeiros gerando confiabilidade para exemplos posteriores, onde as figuras não são conhecidas.

Em sala de aula, o professor pode comentar que, em níveis mais elevados de estudo, temos o curso de Cálculo Diferencial e Integral onde é possível mostrar a convergência para o valor exato da área.

O gráfico dado pela Figura 10 ilustra uma força $(F)$ que é exercida em um móvel durante um deslocamento $(d)$ de 0 a $3 m$, que varia conforme a função $F=\frac{d^{2}}{2}$.

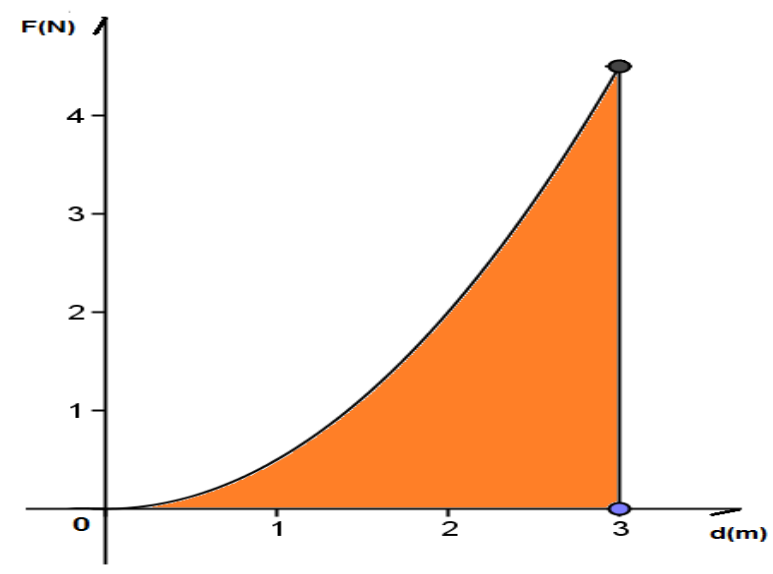

Figura 10: Exemplo de força variável.

Para encontrar o trabalho realizado por essa força, usaremos a mesma estratégia do Exemplo 4.3, ou seja, fazendo aproximações por falta e por excesso já que, no nível médio de ensino, não se conhece uma fórmula direta para o cálculo da área desta figura. 


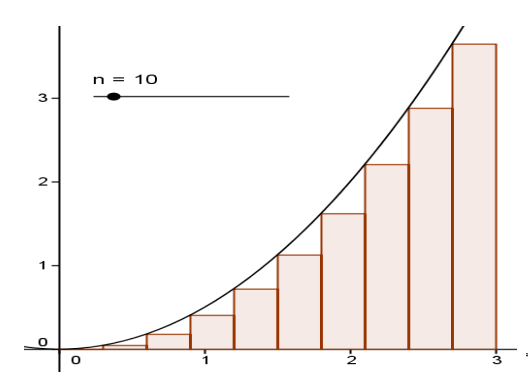

(a) $\mathrm{n}=10$

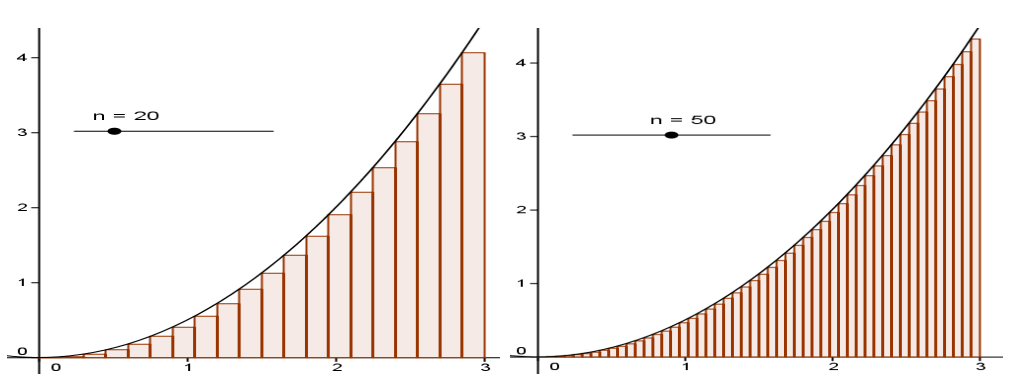

(b) $n=20$

(c) $\mathrm{n}=50$

Figura 11: Área por falta, sob o gráfico da função da Figura 10, para alguns valores de $n$.

\begin{tabular}{|c|c|}
\hline$n$ & $A_{n i}$ \\
\hline 10 & 3,8475 \\
\hline 20 & 4,1681 \\
\hline 50 & 4,3659 \\
\hline 100 & 4,4327 \\
\hline 250 & 4,4730 \\
\hline 530 & 4,4873 \\
\hline 790 & 4,4915 \\
\hline 1000 & 4,4933 \\
\hline
\end{tabular}

Tabela 3: Valores da área por falta, para alguns valores de $n$.

Utilizando no Geogebra o comando "SomaDeRiemannInferior" em conjunto com a ferramenta "Controle Deslizante" foi possível, de forma dinâmica, a perfeita visualização da aproximação, primeiramente por falta, entre a área da soma dos retângulos e a área sob a curva neste intervalo. Outra ferramenta de extrema utilidade para o desenvolvimento desta atividade é "Gravar para a Planilha de Cálculos". Com ela, podemos observar os valores da soma das áreas dos retângulos sendo apresentados numa tabela, conforme variamos a quantidade de subintervalos no Controle Deslizante. O exemplo nos mostra o valor da soma se aproximando de 4,5. Um questionamento que podemos fazer é: este único procedimento para o experimento e seu valor apresentado, pode ser conclusivo? Como o valor 4,5 não ficou explícito, não podemos ter a certeza imediata que este será o valor da área sob a curva. Com isso, direcionamos os alunos a realizar uma segunda etapa do experimento, a aproximação por excesso.

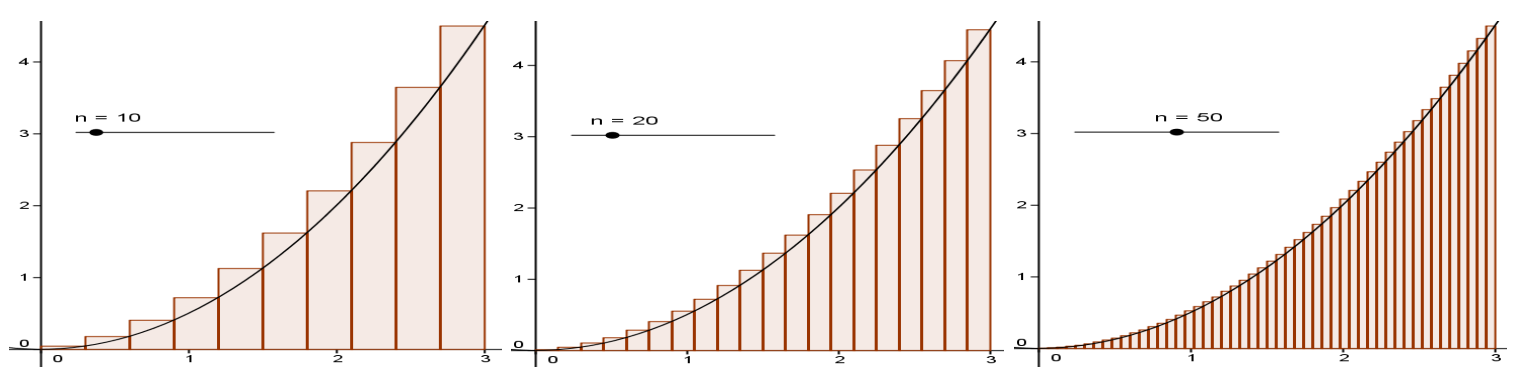

(a) $n=10$

(b) $n=20$

(c) $n=50$

Figura 12: Área por excesso, sob o gráfico da função da Figura 10, para alguns valores de $n$. 


\begin{tabular}{|c|c|}
\hline$n$ & $A_{n s}$ \\
\hline 10 & 4,8431 \\
\hline 20 & 4,7275 \\
\hline 50 & 4,6131 \\
\hline 100 & 4,5615 \\
\hline 250 & 4,5250 \\
\hline 530 & 4,5121 \\
\hline 780 & 4,5081 \\
\hline 990 & 4,5067 \\
\hline
\end{tabular}

Tabela 4: Valores da área por excesso, para alguns valores de $n$.

Neste segundo momento, observamos que a aproximação também nos levou ao valor 4,5. Diante disso, com as aproximações por falta e por excesso convergindo para um mesmo ponto, podemos considerar este valor como sendo da área sob a curva da função neste intervalo. Novamente, este valor pode ser justificado através do Cálculo Integral.

No próximo exemplo, além do desenvolvimento do cálculo da área com aproximação por falta e por excesso, vamos também explorar, na prática, o conceito da Soma de Riemann. Para facilitar a programação, na representação deste último conceito, tomamos, em função do ponto de extremo esquerdo de cada subintervalo, a altura dos retângulos que compõem a soma.

Vamos calcular a área sob o gráfico (Figura 13) da função $f(x)=-x^{2}+4 x$ no intervalo $[0,4]$.

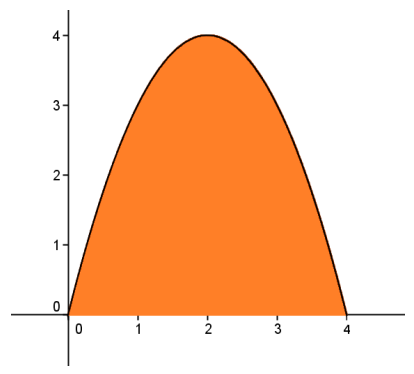

Figura 13: Área sob a parábola.

No desenvolvimento com o Geogebra temos as seguintes representações no cálculo da área através de aproximação por falta:

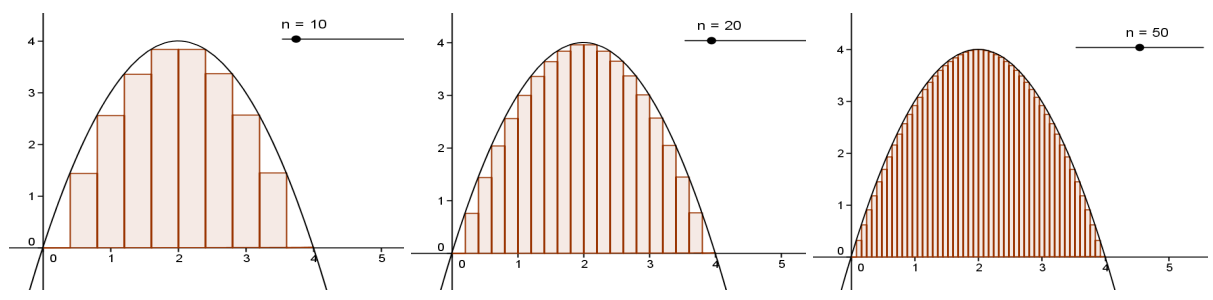
(a) $n=10$
(b) $n=20$
(c) $n=50$

Figura 14: Área, com aproximação por falta, sob o gráfico da função dada pela Figura 13, para alguns valores de $n$. 


\begin{tabular}{|c|c|}
\hline$n$ & $A_{n i}$ \\
\hline 10 & 8,9600 \\
\hline 20 & 9,8400 \\
\hline 60 & 10,3970 \\
\hline 100 & 10,5056 \\
\hline 250 & 10,6025 \\
\hline 530 & 10,6364 \\
\hline 750 & 10,6453 \\
\hline 960 & 10,6495 \\
\hline 10000 & 10,6651 \\
\hline
\end{tabular}

Tabela 5: Valores da área por falta, para alguns valores de $n$.

Vejamos os valores apresentados pelo software na aproximação por excesso.

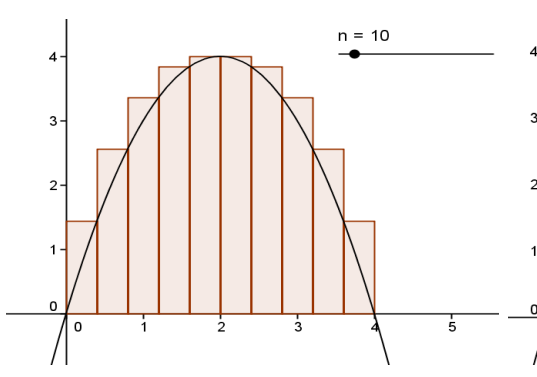

(a) $n=10$

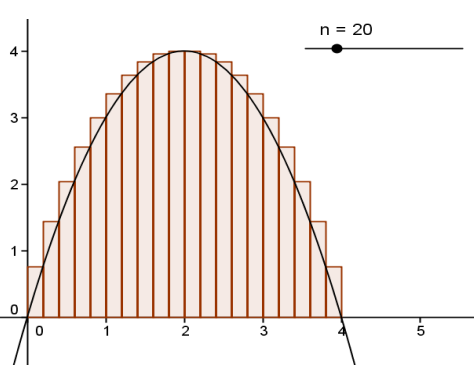

(b) $n=20$

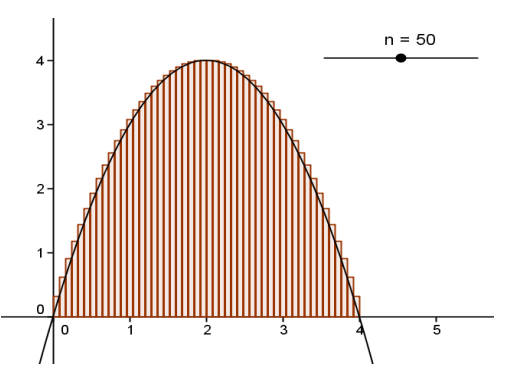

(c) $n=50$

Figura 15: Área, com aproximação por excesso, sob o gráfico da função dada pela Figura 13, para alguns valores de $n$.

\begin{tabular}{|c|c|}
\hline$n$ & $A_{n s}$ \\
\hline 10 & 12,1600 \\
\hline 20 & 11,4400 \\
\hline 60 & 10,9304 \\
\hline 100 & 10,8256 \\
\hline 250 & 10,7305 \\
\hline 530 & 10,6968 \\
\hline 750 & 10,6880 \\
\hline 960 & 10,6833 \\
\hline 10000 & 10,6683 \\
\hline
\end{tabular}

Tabela 6: Valores da área por excesso, para alguns valores de $n$.

Finalmente a representação das aproximações, tomando, em função do ponto de extremo esquerdo de cada intervalo, a referência para a altura de cada retângulo.

Considerando o extremo de cada subintervalo para determinar a altura dos retângulos, este exemplo possui retângulos contidos na região e outros que contém a região que se pretende calcular a área. Vejamos os valores das áreas para alguns valores de $n$. 


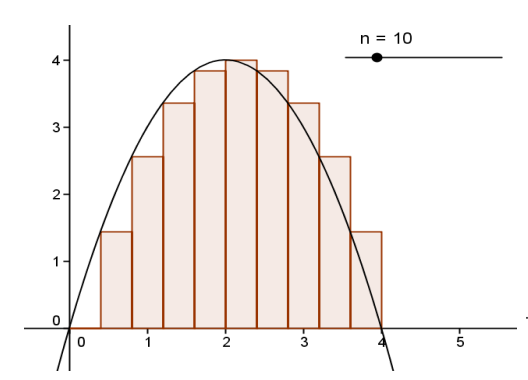

(a) $\mathrm{n}=10$

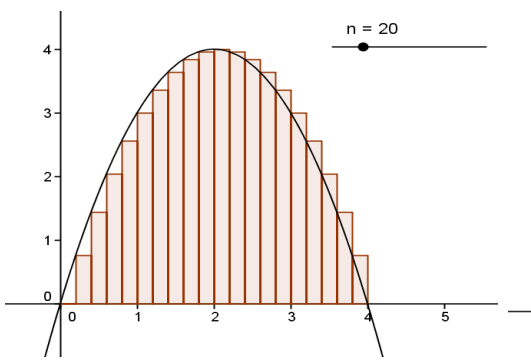

(b) $n=20$

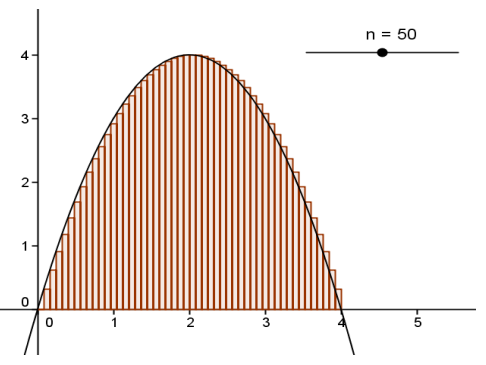

(c) $\mathrm{n}=50$

Figura 16: Área, com o extremo esquerdo como referência, sob o gráfico da função da Figura 13, para alguns valores de $n$.

\begin{tabular}{|c|c|}
\hline$n$ & $A_{n}$ \\
\hline 10 & 10,5600 \\
\hline 20 & 10,6400 \\
\hline 60 & 10,6637 \\
\hline 100 & 10,6656 \\
\hline 250 & 10,6665 \\
\hline 530 & 10,6666 \\
\hline 750 & 10,6666 \\
\hline 960 & 10,6667 \\
\hline
\end{tabular}

Tabela 7: Valores da área com o extremo esquerdo como referência.

Observamos que, em todos os casos, as aproximações convergem ao mesmo valor, possibilitando, de maneira clara e confiável, tirar as conclusões sobre o problema estudado.

Entendemos que, apresentando este exemplo, podemos introduzir de modo intuitivo o conceito da Soma de Riemann de forma adequada ao Ensino Médio, levando o aluno a perceber que a soma converge para o valor da área tomando um valor arbitrário $c_{i}$ dentro de cada intervalo.

As situações já trabalhadas mostraram, de forma detalhada, que com o procedimento proposto conseguimos obter valores que se aproximam do valor da área procurada. Seja por falta, seja por excesso ou ainda, por um extremo, a convergência ocorre.

Os próximos exemplos serão desenvolvidos mostrando apenas uma opção de convergência, simplificando o processo sem perda de confiabilidade. Apresentamos o caso onde a função troca de sinal no intervalo em estudo e o cálculo de área de uma região delimitada pelo gráfico de duas funções.

Para calcular a área $A_{T}$ da região delimitada pelo gráfico da função $f(x)=-(x-1)(x-$ $3)(x-5)$ e o eixo OX, no intervalo [0,5] (Figura 17) escolhemos como referência o extremo esquerdo de cada subintervalo.

Assim, temos: 


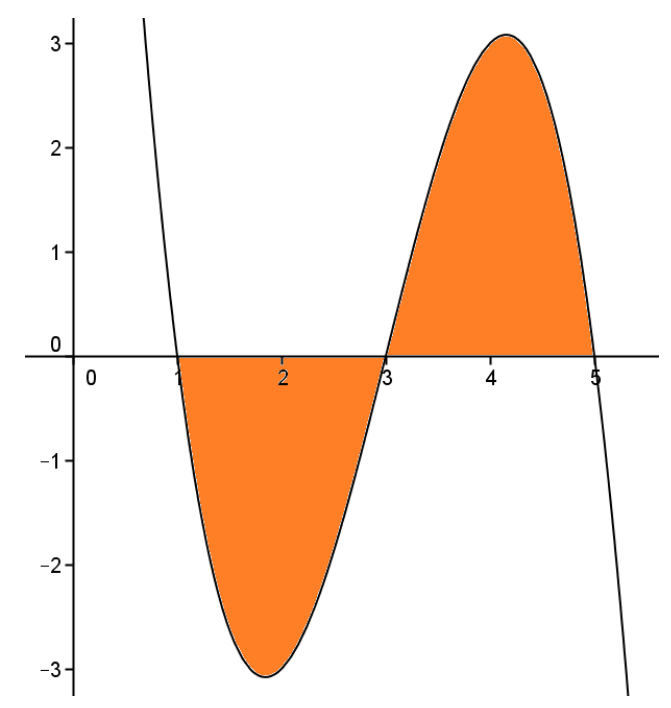

Figura 17: Gráfico de $f(x)=-(x-1)(x-3)(x-5)$.

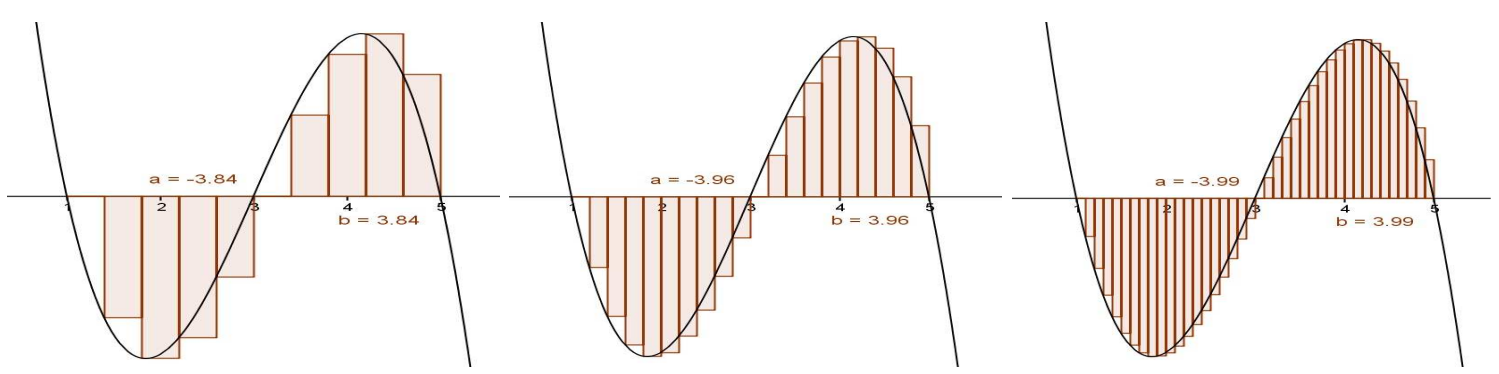

(a) $n=5$

(b) $n=10$

(c) $n=20$

Figura 18: Área sob o gráfico da função da Figura 17, para alguns valores de $n$.

Sendo $A_{1}$ o valor da área da região delimitada no intervalo [1,3] e $A_{2}$ a área da região delimitada no intervalo [3,5], a área $A_{T}$ será a soma das áreas nos dois intervalos. Porém, observamos que para o intervalo $[1,3]$ o Geogebra nos fornece um valor negativo $(A)$ para as aproximações pois $f(x)<0$ neste intervalo (Figura 18). Para que isso seja corrigido, como $A_{1}=-A, A_{T}$ será calculada como:

$$
A_{T}=-A+A_{2} .
$$

Vejamos os valores de $A_{T}$ para alguns valores de $n$.

\begin{tabular}{|c|c|c|c|}
\hline$n$ & $A$ & $A_{2}$ & $A_{T}=-A+A_{2}$ \\
\hline 10 & $-3,9600$ & 3,9600 & 7,9200 \\
\hline 30 & $-3,9952$ & 3,9952 & 7,9905 \\
\hline 50 & $-3,9984$ & 3,9984 & 7,9968 \\
\hline 100 & $-3,9996$ & 3,9996 & 7,9992 \\
\hline 250 & $-3,9999$ & 3,9999 & 7,9999 \\
\hline
\end{tabular}

Tabela 8: Valor da área, para alguns valores de $n$. 
Como último exemplo calcularemos a área $A_{d}$ da região entre os gráficos das funções $f(x)=$ $x^{3} / 2-3 x^{2}+5 x+2$ e $g(x)=3\left(x^{2} / 4\right)-2 x+2$ no intervalo $[0,3]$.

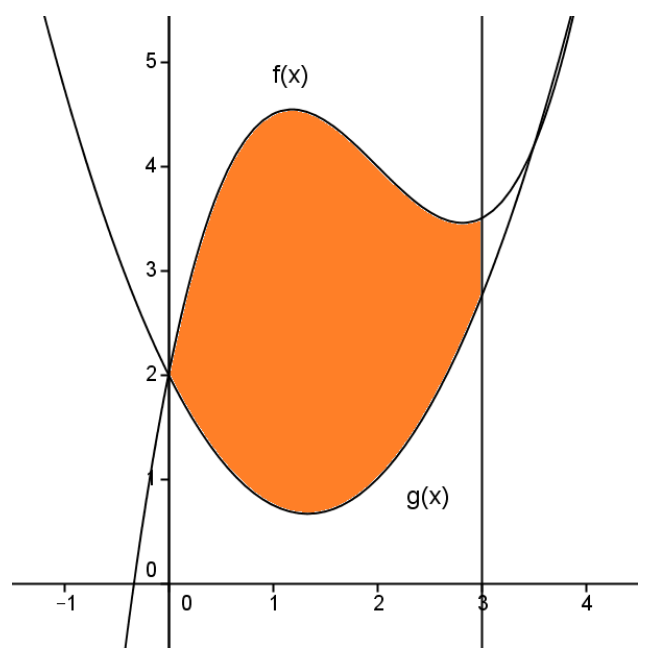

Figura 19: Gráfico de $f(x)$ e $g(x)$.

No modelo da atividade que estamos usando, o Geogebra nos fornece as aproximações das áreas entre os gráficos das funções e o eixo $\mathrm{OX}$.

Para este exemplo, como queremos a área $A_{d}$ da região entre os gráficos das funções no intervalo [0,3], a estratégia será, com aproximações por falta, subtrair da área $A(f(x))$ sob o gráfico da função $f(x)$ que se apresenta na Figura 19, a área $A(g(x))$ sob o gráfico da função $g(x)$ que está abaixo. Esta diferença, seja na representação gráfica, seja nos valores da tabela, representa a área da região.

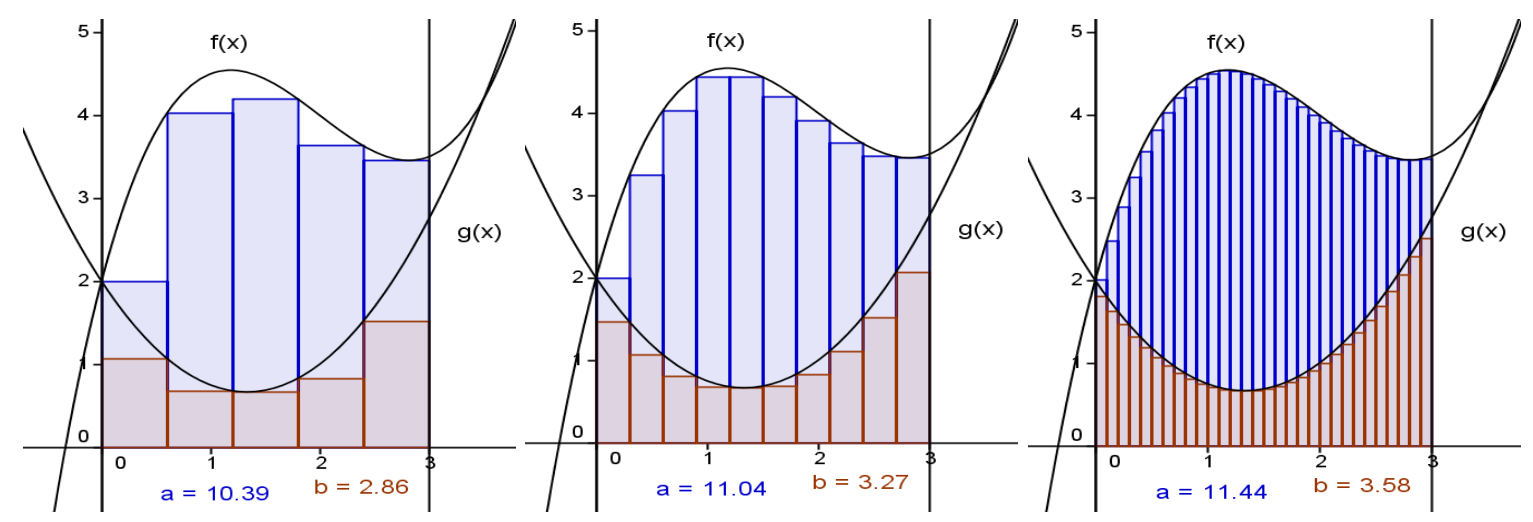

(a) $n=5$

(b) $n=10$

(c) $n=20$

Figura 20: Área entre os gráficos das funções da Figura 19, para alguns valores de $n$.

\section{Considerações Finais}

A utilização do software Geogebra nos exemplos apresentados permite que o aluno calcule área de figuras para as quais ele não tem uma fórmula explícita. Além disso, ele pode visualizar 


\begin{tabular}{|c|c|c|c|}
\hline$n$ & $A(f(x))$ & $A(g(x))$ & $A_{d}=A(f(x))-A(g(x))$ \\
\hline 10 & 11,0398 & 3,2713 & 7,7685 \\
\hline 30 & 11,4374 & 3,5829 & 7,8545 \\
\hline 100 & 11.5695 & 3.6991 & 7,8704 \\
\hline 400 & 11,6112 & 3,7372 & 7,8740 \\
\hline 900 & 11,6189 & 3,7443 & 7,8746 \\
\hline
\end{tabular}

Tabela 9: Valor da área, para alguns valores de $n$.

de forma dinâmica as aproximações obtidas para a área à medida que subdividimos cada vez mais os intervalos. Dessa forma, acreditamos que torna-se viável discutir, a nível de Ensino Médio, o conceito da Soma de Riemann. Este conceito pode ser apresentado de forma intuitiva, levando o aluno a perceber que a soma converge para o valor da área tomando um valor arbitrário $c_{i}$ dentro de cada intervalo. Isto pode contribuir, principalmente, para aqueles alunos que optarem por cursos da área de Ciências Exatas nos estágios seguintes de seus estudos.

\section{Referências}

[1] GUIDORIZZI, H.L. Um Curso de Cálculo. 5. ed. Rio de Janeiro: LTC, 2001. v.1.

[2] LIMA, E.L.: Medida e forma em geometria. Rio de Janeiro: SBM, 2006. v.4.

[3] SIMÕES, A.C. Calculando área sob gráfico de funções. 2014. 45f. Dissertação(Mestrado, em Matemática) - Instituto de Geociências e Ciências Exata, Universidade Estadual Paulista Julio de Mesquita Filho, Rio Claro, 2014. 